\title{
A general, three-dimensional fluid dynamics code for stars in binary systems
}

\author{
Martin E. Beer^ and Philipp Podsiadlowski \\ University of Oxford, Nuclear and Astrophysics Laboratory, Oxford, OX1 3RH, England
}

31 October 2018

\begin{abstract}
We describe the theory and implementation of a three-dimensional fluid dynamics code which we have developed for calculating the surface geometry and circulation currents in the secondaries of interacting binary systems. The main method is based on an Eulerian-Lagrangian scheme to solve the advective and force terms in Euler's equation. Surface normalised spherical polar coordinates are used to allow the accurate modelling of the surface of the star, as is necessary when free surfaces and irradiation effects are to be considered. The potential and its gradient are expressed as sums of Legendre polynomials, which allows a very efficient solution of Poisson's equation. The basic solution scheme, based on operator splitting, is outlined, and standard numerical tests are presented.
\end{abstract}

Key words: binaries: close - X-rays: binaries - hydrodynamics - methods: numerical

\section{INTRODUCTION}

Irradiation of the secondaries in X-ray binaries can dramatically change their appearance and their internal structure. The irradiation pressure force can lead to significant distortions of the surface (Phillips \& Podsiadlowski 2001), while irradiation-driven circulation currents can transport significant amounts of energy to the unirradiated side. There is ample observational evidence for the existence of such circulation currents: e.g. in HZ Her/Her X-1 (Kippenhahn \& Thomas 1979; Schandl, Meyer-Hofmeister \& Meyer 1997), in cataclysmic variables (Davey \& Smith 1992), in Nova Sco during outburst (Shahbaz et al. 2000) and in Cyg X-2 (J. Casares \& P.A. Charles 1999; private communication), there is clear evidence that a substantial amount of X-ray heated material can move beyond the X-ray horizon. Moreover, persistent residuals in the observed radial-velocity curves in $\mathrm{X}$ ray binaries, e.g. in Nova Sco during outburst (Orosz \& Bailyn 1997) and Vela X-1 (Barziv et al. 2001), may provide direct evidence for circulation.

Modelling of the irradiation-induced circulation in binaries is difficult due the three-dimensional nature of the problem. It requires the simultaneous solution of the shape of the irradiated star and the circulation and a proper treatment of the surface boundary conditions.

Attempts to model circulation in irradiated secondaries have been made by various authors in the past, initially using perturbative methods either in planar geometry (Kippenhahn \& Thomas 1979; Kırbıyık \& Smith 1976) or spher-

\footnotetext{
* E-mail: beer@astro.ox.ac.uk
}

ical geometry (Kırbıyık 1982), and more recently nonperturbatively using smooth-particle hydrodynamics (Martin \& Davey 1995). None of these investigations to-date, however, are self-consistent, neither allowing for changes in the surface geometry nor including radiative surface stresses.

On the other hand, circulation currents have been extensively modelled in multi-dimensions in the context of modelling the circulation in the ocean and tidal flows on Earth, where over the years efficient methods have been developed to treat circulation realistically and in a numerically efficient way. A commonly used method is based on an Eulerian-Lagrangian scheme (a description of which may be found in Lu \& Wai 1998). This method is similar to the upwind method but more physically sound in the physical treatment of advective terms and has been shown to be unconditionally stable (Casulli 1990; Casulli \& Cheng 1992).

The purpose of this paper is to present the philosophy and the details of a fairly general three-dimensional fluid dynamics code which we have specially developed for treating the secondaries in interacting binaries, in particular under the influence of external irradiation. The main method is an application of the Eulerian-Lagrangian method by Lu \& Wai (1998) which we have modified for our application, drawing also on the results of related work by Uryū \& Eriguchi (1995, 1996, 1998), Müller \& Steinmetz (1995) and using methods developed in the context of geophysical fluids (see e.g. Pedlosky 1987). At present our code is still somewhat simplified since we do not include the thermodynamic equation, necessitating the use of a polytropic equation of state. In Appendix B, we describe how we plan to generalize the code in the future. In a subsequent paper, we will apply the 
code first to rotation in the standard Roche problems, and then to study irradiation-driven flows in X-ray binaries, considering both the effects of heating and external radiation pressure, and of radiative surface stresses.

The outline of the paper is as follows. In Section we describe the transformations and the usefulness of surface fitting coordinates, Section 3 describes the basic equations and the dimensionless variables used. The theory and implementation of the calculation of the gradient of the gravitational potential is given in Section 4 , including estimates of its accuracy. The general solution method is described in Section 8 . Finally, Sections 6 and 7 present standard numerical tests of the code, advection tests and MacLaurin spheroids.

\section{COORDINATE SYSTEM}

Figure 1 defines the adopted coordinate system, standard spherical polar coordinates centered on the center of mass of the secondary, where the directions of the axes are given by the unit vectors $(\hat{\boldsymbol{r}}, \hat{\boldsymbol{\theta}}, \hat{\boldsymbol{\phi}})$.

\subsection{Surface normalised coordinates}

For our main applications it is important to model the stellar surface accurately and to allow it to adjust freely to satisfy whatever surface boundary conditions are applied. If the outer edge of the coordinate grid did not coincide with the surface, it would be non-trivial to calculate surface stresses and derivatives along the surface accurately. These difficulties can be avoided by using a grid whose boundary is defined by the stellar surface. To achieve this, we follow Uryū \& Eriguchi (1996) and transform our basic equations (see Section B) using spherical surface fitting coordinates, i.e.

$r^{*}=\frac{r}{R(\theta, \phi)}, \quad \theta^{*}=\theta, \quad \phi^{*}=\phi$,

where $R(\theta, \phi)$ is the radius of the star in the direction $(\theta, \phi)$. With this definition, $r^{*}$ is restricted to the range

$0 \leq r^{*} \leq 1$

This means that the grid has to adapt continually as the stellar surface changes and that derivatives are transformed according to:

$$
\begin{aligned}
& R(\theta, \phi) \rightarrow R\left(\theta^{*}, \phi^{*}\right), \\
& \frac{\partial}{\partial r} \rightarrow \frac{1}{R(\theta, \phi)} \frac{\partial}{\partial r^{*}}, \\
& \frac{\partial}{\partial \theta} \rightarrow \frac{\partial}{\partial \theta^{*}}-\frac{r^{*}}{R\left(\theta^{*}, \phi^{*}\right)} \frac{\partial R\left(\theta^{*}, \phi^{*}\right)}{\partial \theta^{*}} \frac{\partial}{\partial r^{*}}, \\
& \frac{\partial}{\partial \phi} \rightarrow \frac{\partial}{\partial \phi^{*}}-\frac{r^{*}}{R\left(\theta^{*}, \phi^{*}\right)} \frac{\partial R\left(\theta^{*}, \phi^{*}\right)}{\partial \phi^{*}} \frac{\partial}{\partial r^{*}} .
\end{aligned}
$$

\subsection{Coordinate grid}

To define the coordinate grid, we split the star into equally spaced intervals in $r, \theta$ and $\phi$ with a total of $N_{\mathrm{r}}, N_{\theta}, N_{\phi}$ elements. The grid points in the $\theta$ and $\phi$ directions are defined by

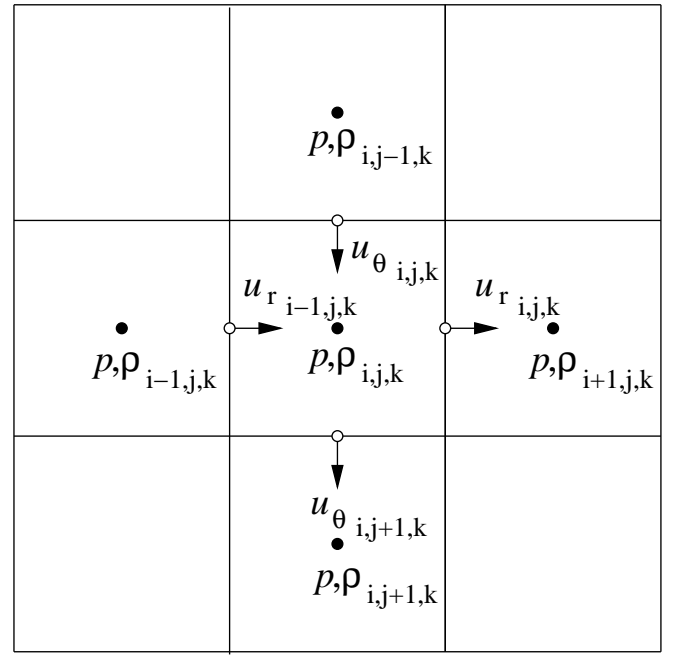

Figure 2. Cross-section of the adopted coordinate grid in the $r$ and $\theta$ directions, indicating where quantities (velocities, $u$, pressure, $p$, and density, $\rho$, are evaluated.

$\theta_{\mathrm{j}}=\frac{\pi(j-0.5)}{2 N_{\theta}}, \quad \phi_{\mathrm{k}}=\frac{2 \pi(k-1)}{N_{\phi}}$,

where we restricted the $\theta$ range assuming even symmetry with respect to the xy-plane, and the surface normalised radial coordinate becomes

$r_{\mathrm{i}, \mathrm{j}, \mathrm{k}}=\frac{i R\left(\theta_{\mathrm{j}}, \phi_{\mathrm{k}}\right)}{N_{\mathrm{r}}}, \quad r_{\mathrm{i}, \mathrm{j}, \mathrm{k}}^{*}=\frac{i}{N_{\mathrm{r}}}$.

Velocities are calculated on cell boundaries whilst pressures and densities are evaluated at the centres of cells. Figure 8 shows a cross-section of the grid indicating where various quantities are evaluated.

\section{BASIC EQUATIONS}

The equation of motion relating velocity $(\boldsymbol{u})$, pressure $(p)$, density $(\rho)$, frictional force $(\mathcal{F})$ and potential $\left(\Phi^{\prime}\right)$ in a frame rotating about the centre of mass of the secondary with angular velocity $\boldsymbol{\Omega}$ is

$\frac{d \boldsymbol{u}}{d t}+2 \boldsymbol{\Omega} \times \boldsymbol{u}=-\frac{1}{\rho} \nabla p-\nabla \Phi^{\prime}+\mathcal{F}$,

where

$\frac{d \boldsymbol{u}}{d t}=\frac{\partial \boldsymbol{u}}{\partial t}+(\boldsymbol{u} \cdot \boldsymbol{\nabla}) \boldsymbol{u}$,

and the potential term is given by

$\Phi^{\prime}=\Phi+\frac{G M_{1} r \sin \theta \cos \phi}{a^{2}}-\frac{(\boldsymbol{\Omega} \times \boldsymbol{r})^{2}}{2}-\frac{G M_{1}}{r_{2}}$.

Here $r_{2}$ is the distance from the primary to the point $(r, \theta, \phi)$, and the potential of the secondary $\Phi$ is given by Poisson's equation

$\nabla^{2} \Phi=4 \pi G \rho$.

The continuity equation,

$\frac{d \rho}{d t}+\rho \nabla \cdot \boldsymbol{u}=0$ 


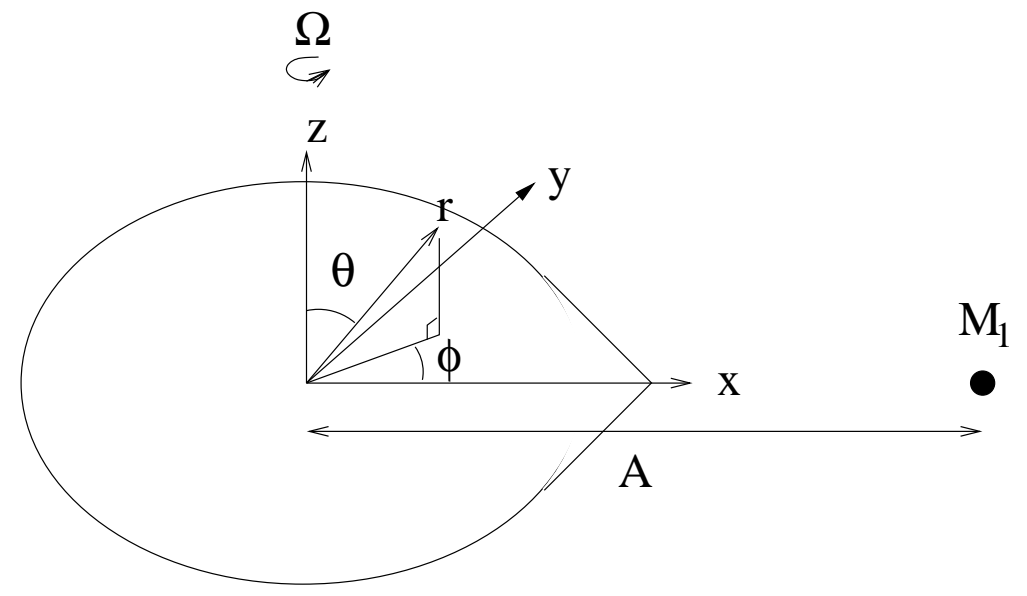

Figure 1. Schematic diagram defining the coordinate systems $(r, \theta, \phi)$ and $(x, y, z)$ relative to the system geometry and other binary parameters ( $A$ : orbital separation, $M_{1}$ : mass of the primary, $\boldsymbol{\Omega}$ : angular velocity of the secondary).

can be written in integral form, using the divergence theorem, as

$\frac{\partial}{\partial t} \int_{V} \rho d V+\int_{d V} \rho \boldsymbol{u} \cdot \hat{\boldsymbol{n}} d S=0$

where $\hat{\boldsymbol{n}}$ is a unit surface normal vector. These equations need to be solved along with an equation of state, assumed to be a polytropic equation of state at present which relates pressure and density according to

$p=K \rho^{\left(1+\frac{1}{n}\right)}$,

where $K$ and $n$ are the polytropic constant and polytropic index, respectively.

\subsection{Boundary conditions}

To completely specify the mathematical problem, we also need to specify a set of boundary conditions at the centre and the surface. The central boundary conditions are unproblematic and are given by

$\boldsymbol{u}=0, \quad \frac{\partial u_{\mathrm{r}}}{\partial r}=0, \quad \rho=\rho_{\mathrm{c}}$.

The simplest boundary condition for the surface are the zero-pressure condition and the conservation of the mass of the secondary,

$p=0, \quad M_{2}=$ constant,

or, more realistically, a pressure condition that assumes an atmosphere in hydrostatic equilibrium, i.e.

$p=\frac{2 g}{3 \kappa}$,

where $g$ is the effective surface gravity, and $\kappa$ the photospheric value of the opacity (see e.g. Kippenhahn \& Weigert 1994).

In the case of irradiated stars, a special treatment of the surface layer is required since the irradiation flux is deposited in a thin (turbulent) surface layer (of order an atmospheric scale height), which will generally be smaller than our grid size. A separate atmosphere calculation, which includes the effects of heating and irradiation pressure, then determines the pressure across the surface of the star (analogously to the case of normal stars; also see Tout et al. 1989). In addition, the variation of the radiation pressure force causes a surface stress which drives horizontal motion perpendicular to the stress and vertical motion in a thin turbulent boundary layer (an 'Ekman' layer), a process known as 'Ekman' pumping. This produces a vertical velocity component in regions where the surface stress varies. This process is entirely analogous to the wind-driven circulation in oceanic circulation systems (see chapter 5 of Pedlosky 1987) and can be treated analogously.

\subsection{Surface adjustment}

At the end of each time step, the surface is adjusted using the current values of the velocity at the free surface. The surface normal is calculated by taking the cross product of adjacent surface points

$\hat{\boldsymbol{n}}_{\mathrm{j}, \mathrm{k}}=\left(\boldsymbol{R}_{\mathrm{j}+1, \mathrm{k}}-\boldsymbol{R}_{\mathrm{j}-1, \mathrm{k}}\right) \times\left(\boldsymbol{R}_{\mathrm{j}, \mathrm{k}+1}-\boldsymbol{R}_{\mathrm{j}, \mathrm{k}-1}\right)$,

unless $k=1, j=N_{\theta}$ and $L_{\mathrm{x}}>0$, in which case

$\hat{\boldsymbol{n}}_{\mathrm{j}, \mathrm{k}}=\left(\boldsymbol{R}_{\mathrm{j}, \mathrm{k}}-\boldsymbol{R}_{\mathrm{j}-1, \mathrm{k}}\right) \times\left(\boldsymbol{R}_{\mathrm{j}, \mathrm{k}+1}-\boldsymbol{R}_{\mathrm{j}, \mathrm{k}-1}\right)$.

This second condition is necessary in cases where this point corresponds to a conical critical point, as e.g. for the inner Lagrangian point $L_{1}$ (see Phillips \& Podsiadlowski 2001 for discussion). The dot product of the surface velocity with the surface normal gives the velocity component normal to the surface. Equating this to the component of the surface adjustment in the radial direction $(\delta r)$, which is normal to the surface, yields

$\delta r=\frac{\boldsymbol{u} \cdot \hat{\boldsymbol{n}}}{\hat{\boldsymbol{n}} \cdot \hat{\boldsymbol{r}}}$.

This is added to the current value of $r$ at the end of each time step. In order to ensure mass conservation, the density of each $\theta, \phi$ element is scaled by the ratio of the old and new radii cubed

$\rho \rightarrow\left(\frac{r}{r+\delta r}\right)^{3} \rho$.

This ensures that the density decreases as the radius increases, conserving mass in the process. The central density 
is calculated by extrapolating the densities in the central regions of the star. It is only used as a boundary condition in the potential calculation (Section 4).

\subsection{Viscosity}

Artificial viscosity is often added in numerical simulations to smooth out the flow and to broaden shock fronts. Stone \& Norman (1992) in their code ZEUS-2D use an artificial pressure similar to that of von Neumann \& Richtmeyer (1950):

$q= \begin{cases}C^{2} \rho(\Delta v)^{2} & \text { if }(\Delta v)<0, \\ 0 & \text { otherwise },\end{cases}$

where $\Delta v$ is the change in velocity across a cell and $C=$ $l / \Delta \mathrm{x}$. $C$ measures the number of zones over which the artificial viscosity spreads a shock and is typically chosen to be $C \sim 3$. They also consider an artificial linear viscous pressure,

$q=C_{1} \rho C_{a} \Delta v$,

to damp instabilities in stagnant regions of the flow, where $C_{1}$ is a constant of order unity and $C_{a}$ is the adiabatic sound speed. This is calculated separately for each direction and then added, e.g. in the $r$ direction as

$\hat{\boldsymbol{r}} \cdot \mathcal{F}=-\frac{1}{\rho} \frac{\partial q_{\mathrm{r}}}{\partial r}$

\subsection{Dimensionless variables}

In the code we use dimensionless variables defined as:

$\tilde{R}=\frac{R}{R_{\mathrm{s}}}, \quad \tilde{\rho}=\frac{\rho}{\rho_{\mathrm{c}}}, \quad \tilde{\nabla}=R_{\mathrm{s}} \nabla, \quad \tilde{p}=\frac{p}{G \rho_{\mathrm{c}}{ }^{2} R_{\mathrm{s}}{ }^{2}}$,

$\tilde{\Phi}=\frac{\Phi}{G \rho_{\mathrm{c}} R_{\mathrm{s}}^{2}}, \quad \tilde{\boldsymbol{u}}=\frac{\boldsymbol{u}}{R_{\mathrm{s}} \sqrt{G \rho_{\mathrm{c}}}}, \quad \tilde{\Omega}=\frac{\Omega}{\sqrt{G \rho_{\mathrm{c}}}}$,

$\tilde{M}=\frac{M}{\rho_{\mathrm{c}} R_{\mathrm{s}}^{3}}, \quad \tilde{L_{\mathrm{x}}}=\frac{L_{\mathrm{x}}}{G R_{\mathrm{s}}{ }^{2} c}, \quad \tilde{t}=t \sqrt{G \rho_{\mathrm{c}}}$,

$\tilde{\mathcal{F}}=\frac{\mathcal{F}}{G \rho_{\mathrm{c}} R_{\mathrm{s}}}, \quad \tilde{\nu}=\frac{\nu}{R_{\mathrm{s}}{ }^{2} \sqrt{G \rho_{\mathrm{c}}}}, \quad \tilde{\kappa}=\kappa \rho_{\mathrm{c}} R_{\mathrm{s}}$,

where $R_{\mathrm{s}}$ is the solar radius, $\rho_{\mathrm{c}}$ the initial central density and $G$ the gravitational constant. This transforms equations (9), (14) \& 15) to:

$\frac{d \tilde{\boldsymbol{u}}}{d \tilde{t}}+2 \tilde{\boldsymbol{\Omega}} \times \tilde{\boldsymbol{u}}=-\frac{1}{\tilde{\rho}} \tilde{\nabla} \tilde{p}-\tilde{\nabla} \tilde{\Phi}^{\prime}+\tilde{\mathcal{F}}$,

$\frac{\partial}{\partial \tilde{t}} \int_{\tilde{V}} \tilde{\rho} d \tilde{V}+\int_{d \tilde{V}} \tilde{\rho} \tilde{\boldsymbol{u}} \cdot \hat{\boldsymbol{n}} d \tilde{S}=0$,

$\tilde{p}=\frac{K \tilde{\rho}^{\left(1+\frac{1}{n}\right)}}{G R_{\mathrm{s}}^{2} \rho_{\mathrm{c}}\left(1-\frac{1}{n}\right)}$.

\section{POTENTIAL CALCULATION}

\subsection{Theory}

Müller \& Steinmetz (1995) developed an efficient algorithm for solving Poisson's equation which utilizes spherical coordinates and an expansion into spherical harmonics. This results in an algorithm which has a computational cost proportional to $(L+1)^{2} N$ where $L$ is the highest order harmonic considered and $N$ the total number of grid points. The general solution of Poisson's equation in spherical harmonics, $Y^{l m}(\theta, \phi)$ (Morse \& Feshbach 1953) can be written as

$$
\begin{aligned}
& \Phi(r, \theta, \phi)=-G \sum_{l=0}^{\infty} \frac{4 \pi}{2 l+1} \sum_{m=-l}^{l} Y^{l m}(\theta, \phi) \times \\
& {\left[\frac{1}{r^{l+1}} C^{l m}(r)+r^{l} D^{l m}(r)\right], }
\end{aligned}
$$

where

$$
\begin{aligned}
& C^{l m}(r)=\int_{4 \pi} d \Omega^{\prime} Y^{l m *}\left(\theta^{\prime}, \phi^{\prime}\right) \int_{0}^{r} d r^{\prime} r^{\prime+2} \rho\left(r^{\prime}, \theta^{\prime}, \phi^{\prime}\right) \\
& D^{l m}(r)=\int_{4 \pi} d \Omega^{\prime} Y^{l m *}\left(\theta^{\prime}, \phi^{\prime}\right) \int_{r}^{\infty} d r^{\prime} r^{\prime-l} \rho\left(r^{\prime}, \theta^{\prime}, \phi^{\prime}\right), \\
& d \Omega^{\prime}=\sin \theta^{\prime} d \theta^{\prime} d \phi^{\prime} .
\end{aligned}
$$

By differentiating this equation we may obtain analytical formulae for for the gradient of the potential in spherical coordinates (as derived in Appendix C1):

$$
\begin{aligned}
& \frac{\partial \Phi(r, \theta, \phi)}{\partial r}=-G \sum_{l=0}^{\infty} \frac{4 \pi}{2 l+1} \sum_{m=-l}^{l} Y^{l m}(\theta, \phi) \times \\
& {\left[\frac{-(l+1)}{r^{l+2}} C^{l m}(r)+l r^{l-1} D^{l m}(r)\right] .} \\
& \frac{\partial \Phi(r, \theta, \phi)}{\partial \theta}=-G \sum_{l=0}^{\infty} \frac{4 \pi}{2 l+1} \sum_{m=-l}^{l} Y^{l m}(\theta, \phi) \times \\
& {\left[\frac{P_{l}^{|m+1|}(\cos \theta)}{P_{l}^{|m|}(\cos \theta)}+\frac{m \cos \theta}{\sin \theta}\right]\left[\frac{1}{r^{l+1}} C^{l m}(r)+r^{l} D^{l m}(r)\right] .} \\
& \frac{\partial \Phi(r, \theta, \phi)}{\partial \phi}=-G \sum_{l=0}^{\infty} \frac{4 \pi}{2 l+1} \sum_{m=-l}^{l} i m Y^{l m}(\theta, \phi) \times \\
& {\left[\frac{1}{r^{l+1}} C^{l m}(r)+r^{l} D^{l m}(r)\right] .}
\end{aligned}
$$

For odd $l+m, P_{l}^{m}\left(\cos \theta^{\prime}\right)$ is an odd function in $\theta^{\prime}$. Since the density is an even function w.r.t. $\theta^{\prime}$ (because of the assumed symmetry with respect to the $x y$-plane) $\rho\left(\theta^{\prime}, \phi^{\prime}, r^{\prime}\right) \sin \theta^{\prime} P_{l}^{m}\left(\cos \theta^{\prime}\right)$ is an odd function and its integral from $\theta=0$ to $\pi$ in equations (31) \& 32 is zero. Hence terms odd in $l+m$ do not contribute to the sum, and we only need to include values of $m$ from 0 to $l$ and double the contribution of the positive $m$ term.

In our code we deal with $\Phi^{\prime}$ rather than $\Phi$,

$$
\begin{aligned}
\Phi^{\prime}(r, \theta, \phi)= & (r, \theta, \phi)- \\
& \frac{(\Omega r \sin \theta)^{2}}{2}+\frac{G M_{1} r \sin \theta \cos \phi}{a^{2}}-\frac{G M_{1}}{r_{2}},
\end{aligned}
$$

where $a$ is the separation of the binary system and $r_{2}$ the distance from the primary to the point $(r, \theta, \phi)$,

$r_{2}=\sqrt{a^{2}+r^{2}-2 a r \sin \theta \cos \phi}$.

This then yields:

$\frac{\partial \Phi^{\prime}}{\partial r}=\frac{\partial \Phi}{\partial r}-r \Omega^{2} \sin ^{2} \theta+$ 


$$
\frac{G M_{1} \sin \theta \cos \phi}{a^{2}}+\frac{G M_{1}(r-a \sin \theta \cos \phi)}{\left(a^{2}+r^{2}-2 a r \sin \theta \cos \phi\right)^{\frac{3}{2}}}
$$

$$
\begin{aligned}
\frac{\partial \Phi^{\prime}}{\partial \theta}= & \frac{\partial \Phi}{\partial \theta}-(r \Omega)^{2} \sin \theta \cos \theta+ \\
& \frac{G M_{1} r \cos \theta \cos \phi}{a^{2}}-\frac{G M_{1} a r \cos \theta \cos \phi}{\left(a^{2}+r^{2}-2 a r \sin \theta \cos \phi\right)^{\frac{3}{2}}},
\end{aligned}
$$

$$
\begin{aligned}
\frac{\partial \Phi^{\prime}}{\partial \phi}= & \frac{\partial \Phi}{\partial \phi}- \\
& \frac{G M_{1} r \sin \theta \sin \phi}{a^{2}}-\frac{G M_{1} a r \sin \theta \sin \phi}{\left(a^{2}+r^{2}-2 a r \sin \theta \cos \phi\right)^{\frac{3}{2}}}
\end{aligned}
$$

\subsection{Accuracy}

In Appendix C2, we describe the implementation of this potential calculation in the code. To test the accuracy of the potential calculation, we compared it to three simple cases in which analytical solutions exist. We also compared it to the ZEUS-2D code (Stone \& Norman 1992) for which the same tests have been performed. For the purposes of comparison, we chose their test cases in spherical polar coordinates in which they assumed equatorial symmetry as in our calculations. They do not list the number of grid points they used. Here we used $50 \times 48 \times 48$ grid points. Table 1 shows the comparison in the potential calculation for three cases of a homogeneous sphere, a centrally condensed sphere and a homogeneous ellipsoid.

Table 1 shows that unlike the calculation of ZEUS-2D the error in the gradient of the potential is independent of the density distribution i.e. the errors are similar for both the homogeneous sphere and the centrally condensed sphere. We have also verified that increasing the $\theta$ resolution decreases the error in the calculation. The error in the calculation for the homogeneous ellipsoid is smaller than that of the ZEUS-2D calculation. Indeed, it is only large at the surface - throughout the remainder of the star it remains of similar order as in the calculations with spherical geometries. We have also calculated the error in the $\theta$ derivative of the potential. We find that this is of similar order to the error in the $r$ direction.

\section{SOLUTION METHOD}

\subsection{Operator splitting of the equation of motion}

The equation of motion is solved in two parts using the method of operator splitting or fractional step. With this technique the equation of motion is split into two parts. One representing the advective terms, and another representing the force terms. The advantage of this method is that different numerical techniques may be used to solve the two equations representing physically different processes.

We split equation (9) into two parts, one containing the advective terms, the other the force terms. Once the first part is solved, its solution is used in the second part to find the solution corresponding to the full time step. Representing the time derivative of these equations in finite difference form yields

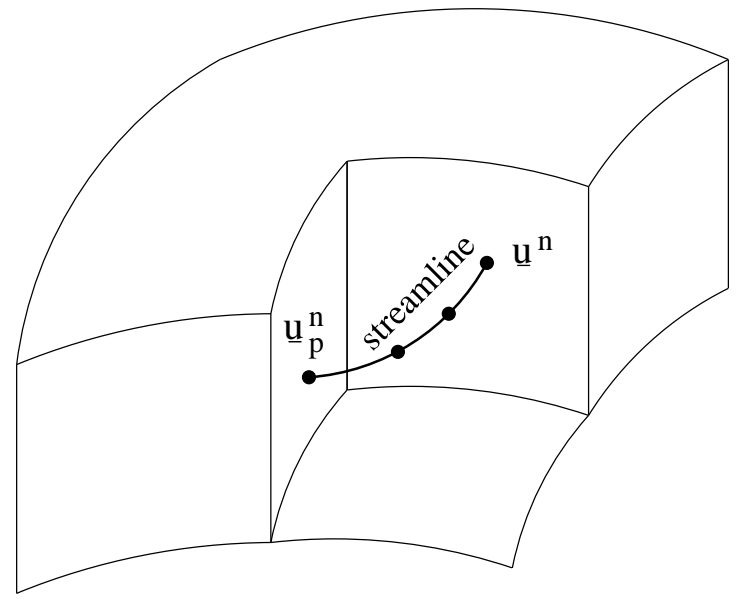

Figure 3. Diagram showing the path interpolation used for the Eulerian-Lagrangian method.

$$
\begin{aligned}
& \frac{\boldsymbol{u}^{a}-\boldsymbol{u}_{\mathrm{p}}^{n}}{\delta t}+2 \boldsymbol{\Omega} \times \boldsymbol{u}^{a}=0, \\
& \frac{\boldsymbol{u}^{n+1}-\boldsymbol{u}^{a}}{\delta t}=-\frac{1}{\rho} \nabla p-\nabla \Phi^{\prime}+\mathcal{F},
\end{aligned}
$$

where we have followed the notation of Lu \& Wai (1998), and the subscript $\mathrm{p}$ refers to the position of the element of interest in the previous time step. Each part of the equation is solved for a full time step $(\delta t)$, and we have used the notation $\boldsymbol{u}^{a}$ to indicate the solution for the velocity once the first equation, containing the advective terms, has been solved.

By solving the equations using the velocity of the fluid element from the previous time step, the non-linear term $(\boldsymbol{u} \cdot \boldsymbol{\nabla}) \boldsymbol{u}$ is eliminated from the equations. This means that advective terms in the equations are solved in a Lagrangian frame (for fixed mass elements) rather than an Eulerian frame (with fixed positions) where the remainder of the terms are solved for. Hence this method is known as an Eulerian-Lagrangian method. Figure 3 illustrates how $\boldsymbol{u}_{\mathrm{p}}^{n}$ is calculated. The figure shows a cut away element of the fluid. Using the velocity $\boldsymbol{u}^{n}$, we calculate the position of the fluid element at a time step $\delta t$ before the present one. Interpolating the velocity grid yields the velocity $\boldsymbol{u}_{\mathrm{p}}^{n}$ at this position. In actuality, we split the time step into a number of substeps enabling an accurate calculation of the streamlines of the fluid.

\subsection{Solution of the continuity equation on an adaptive grid}

The grid used in the code is adaptive. At the end of each time step, the grid is rescaled so that its boundary represents the surface of the star. Thus, the mass within each cell will change at the end of each time step. To compensate for this in solving the integral form of the continuity equation (equation 14), the surface velocity is calculated at each iteration of the continuity equation and the radial velocities used in the calculation are relative to this

$u_{r}^{\prime}(\theta, \phi)=u_{r}(\theta, \phi)-\frac{\left.u_{r}(\theta, \phi)\right|_{\mathrm{s}} i}{N_{\mathrm{r}}}$ 


\begin{tabular}{lcc}
\hline Object & Max. error in $\frac{\partial \Phi}{\partial r}$ & Max. error in $\nabla \Phi$ in ZEUS-2D \\
\hline Homogeneous Sphere & $1.40 \times 10^{-1} \%$ & $1.11 \times 10^{-2} \%$ \\
Centrally Condensed Sphere & $1.72 \times 10^{-1} \%$ & $9.98 \times 10^{-2} \%$ \\
Homogeneous Ellipsoid & $1.41 \%$ & $1.86 \%$ \\
\hline
\end{tabular}

Table 1. A comparison of the potential calculation in our code, performed on a three-dimensional grid, to the two-dimensional calculations using the ZEUS-2D code for three simple cases with analytic solutions.

where the prime indicates the velocity used in the calculation and the subscript s a quantity evaluated at the surface. Dorfi shows in LeVeque et al. (1998) [p. 279] that time centering of the equations results in second order accuracy in $\delta t$. Hence the variables are evaluated at half-odd-integer time steps i.e. when calculating the mass flow through a cell boundary we use $\boldsymbol{u}^{n+\frac{1}{2}}$ and $\rho^{n+\frac{1}{2}}$.

\subsection{Extrapolation of the pressure gradient to the surface}

The radial velocity needs to be calculated at the surface which would require a ghost point for pressure half a radial grid zone past the surface. Instead we use the surface pressure which is one of the boundary conditions. Thus we evaluate the pressure gradient one quarter of a zone below the surface. However, this still yields an incorrect value for the surface pressure gradient, so that in equilibrium it will not balance the potential gradient causing a spurious surface velocity. To correct for this, we may extrapolate the pressure gradient to the surface. We find that the most accurate balance occurs when we do a cubic extrapolation of the points immediately below the surface (including one quarter of a zone from the surface). This is the method we adopted in the code.

\subsection{Iteration procedure}

Figure 4 shows the iteration procedure used in the code. After initialization the main loop of the code is entered. The Eulerian-Lagrangian method is used to find the solution of the advective terms. A sub loop is then entered in which the velocities and density are solved. Using the current values of pressure and density, the new values for the velocities are calculated. With these velocities the new densities can be determined. Once these have converged, the end of a time step has been reached and the surface is adjusted to fit the new shape of the star. Overall convergence is then tested until a solution is found which is written to an output file.

\section{ADVECTION TESTS}

To test the advection in our code, we use two simple tests. Both are in spherical geometry and have also been carried out by Stone \& Norman (1992) for their code ZEUS-2D to which we compare it.

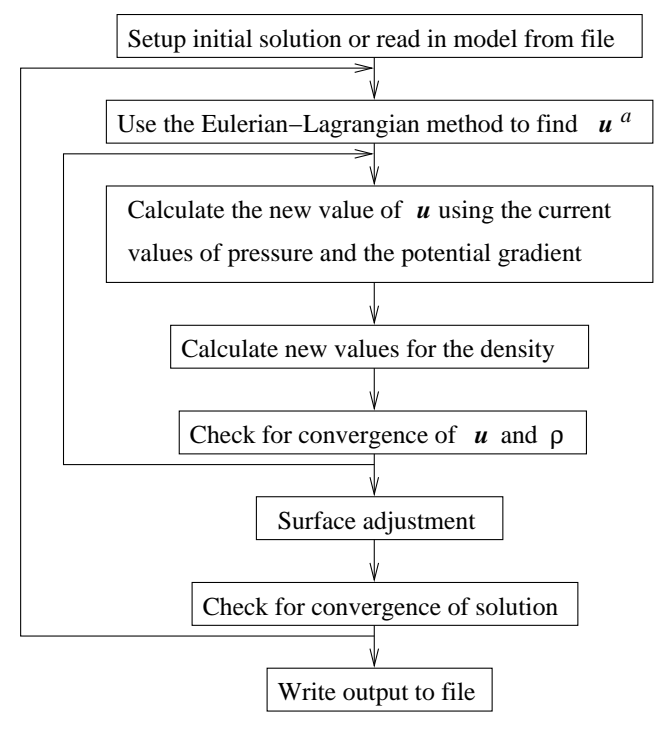

Figure 4. A flow chart diagram showing the iteration procedure used in the code

\begin{tabular}{ccc}
\hline Time step & Density at $t=4$ & Error in the density at $t=4$ \\
\hline $10^{-2}$ & $6.522 \times 10^{-6}$ & $6.14 \%$ \\
$10^{-3}$ & $6.181 \times 10^{-6}$ & $0.60 \%$ \\
$10^{-4}$ & $6.148 \times 10^{-6}$ & $0.06 \%$ \\
\hline
\end{tabular}

Table 2. A comparison of the calculated density to the analytical solution for the expansion of a homogeneous sphere in a velocity field.

\subsection{Expansion of a homogeneous sphere in a velocity field}

The first test is a "relaxation" problem for a pressure-free and gravity-free homogeneous gas with a velocity field proportional to the radius $\left(u_{\mathrm{r}}=v_{0} r\right)$. The density will decay exponentially with time as

$\rho(t)=\rho_{0} e^{-3 v_{0} t}$.

At $v_{0} t=4$, the density should have decayed by almost six orders of magnitude to $\rho=6.144 \times 10^{-6} \rho_{0}$. In our calculation we use $v_{0}=1, \rho_{0}=1$ and three time steps of $10^{-2}, 10^{-3}$ and $10^{-4}$. The results are shown in Table 2 .

At $t=4$ our code is in better agreement than ZEUS$2 \mathrm{D}\left(5.60 \times 10^{-6}, 8.8 \%\right)$ for all the time steps considered, the error in the density being directly proportional to the time step considered. Stone \& Norman (1992), however, provide 


\begin{tabular}{cc}
\hline Time step & Max. error in density at $t=0.535$ \\
\hline $10^{-3}$ & $5.30 \%$ \\
$10^{-4}$ & $0.50 \%$ \\
\hline
\end{tabular}

Table 3. A comparison of density calculated in the code to the analytical solution for the collapse of a pressure-free sphere.

no information on the time step they use, so this cannot be compared further.

\subsection{Pressure-free collapse of a sphere under gravity}

The second advection test we use is the collapse of a homogeneous, pressure-free sphere under gravity. Hunter (1962) showed that

$\frac{r}{r_{0}}=\cos ^{2} \beta, \quad \frac{\rho}{\rho_{0}}=\cos ^{-6} \beta$,

where

$\beta+\frac{\sin 2 \beta}{2}=t \sqrt{\frac{8 \pi G \rho_{0}}{3}}$,

and $r_{0}$ and $\rho_{0}$ are the initial values of the radius and the density, respectively. For $r_{0}=\rho_{0}=G=1$, the free-fall time (the time at which the sphere has collapsed to a point) is 0.543 . The test was ran at two different time steps $\left(10^{-3}\right.$ and $\left.10^{-4}\right)$, and the density, radius and the mass were compared to the analytical solution and the results obtained with ZEUS-2D.

Table 3 shows the errors in the calculated density in comparison to the analytical solution. In all cases, the density profile remained flat and the mass constant. This demonstrates the ability of the code to adjust the surface of the star correctly. Figure 5 shows a comparison at time $t=$ 0.535 with the analytical solution for a time step of $10^{-4}$. Note how the grid is only defined within the sphere allowing an accurate representation of the problem with no spurious points exterior to the surface. In the calculation with ZEUS$2 \mathrm{D}$, a number of grid points end up outside the surface - a direct consequence of their use of ghost zones beyond the boundary.

\section{COMPARISON TO LAI, RASIO \& SHAPIRO}

\subsection{The compressible Maclaurin sequence}

Lai, Rasio \& Shapiro (1993, hereafter LRS) calculated a sequence of compressible Maclaurin spheroids based on an energy variational method. In order to compare our code with their calculations, we require the angular momentum $(J)$, kinetic energy $(T)$ and potential energy $(W)$ :

$J=I \Omega+\int_{V} \rho r \sin \theta u_{\phi} d V$

$T=\frac{1}{2} I \Omega^{2}+\int_{V} \frac{1}{2} \rho\left(u_{r}^{2}+u_{\theta}^{2}+u_{\phi}^{2}\right) d V$

$W=\frac{1}{2} \int_{V} \rho \Phi d V$

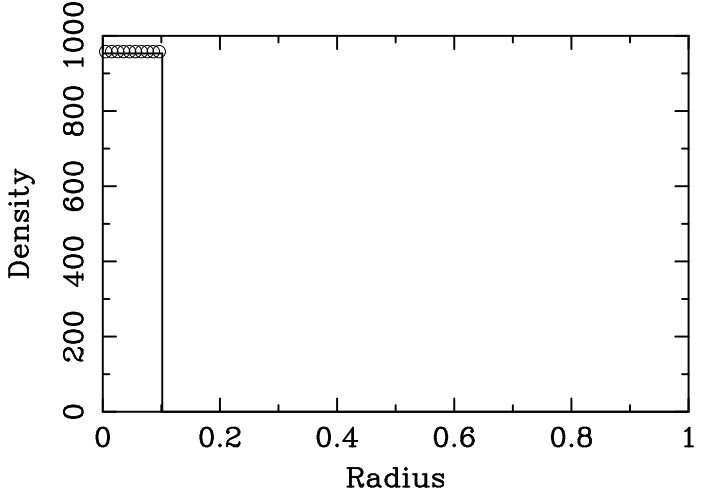

Figure 5. Test simulation for the pressure-free collapse of a homogeneous sphere under gravity. The comparison shows the density as a function of radius at dimensionless time $t=0.535$ for a time step of $10^{-4}$. The solid curves represent the analytical solution and the open circles our model results.

where $I$ is the moment of inertia of the star,

$I=\int_{V} \rho r^{2} \sin ^{2} \theta d V$

These parameters can be brought into dimensionless forms using the dimensional scalings:

$J \equiv(\tilde{J}+\tilde{I} \tilde{\Omega}) R_{\mathrm{s}}{ }^{5} \sqrt{G \rho_{\mathrm{c}}{ }^{3}}$,

$T \equiv\left(\tilde{T}+\frac{1}{2} \tilde{I} \tilde{\Omega}^{2}\right) G \rho_{\mathrm{c}}{ }^{2} R_{\mathrm{s}}{ }^{5}$,

$W \equiv \tilde{W} G \rho_{\mathrm{c}}^{2} R_{\mathrm{s}}{ }^{5}$.

LRS calculates universal dimensionless quantities that are functions of the eccentricity of the spheroid only. The eccentricity is defined as

$e=\sqrt{1-\frac{R_{\text {pole }}^{2}}{R_{\text {equator }}^{2}}}$.

Transforming into the dimensionless units used in our code, their equation (3.27) becomes

$\hat{\Omega}^{2} \equiv \kappa_{n}(1-n / 5)\left(\frac{\tilde{\Omega}^{2} \rho_{\mathrm{c}}}{\pi \bar{\rho}}\right)$,

$\hat{J}^{2} \equiv \frac{1}{\kappa_{n}}(1-n / 5)\left[\frac{(\tilde{J}+\tilde{I} \tilde{\Omega})^{2} R_{\mathrm{s}}}{\tilde{M}^{3} \bar{R}}\right]$,

$\hat{W} \equiv \frac{5-n}{3}\left(\frac{\tilde{W} \bar{R}}{\tilde{M}^{2} R_{\mathrm{s}}}\right)$

$\hat{R} \equiv\left(\frac{\bar{R}}{R_{0}}\right)^{\frac{3-n}{n}}$,

where

$\bar{R}=\left(R_{\text {pole }} R_{\text {equator }}^{2}\right)^{\frac{1}{3}}$,

is the mean value of the radius of the star (where $R_{\text {pole }}$ and $R_{\text {equator }}$ are the radii of the pole and equator respectively), $R_{0}$ is the radius of a spherical polytrope of mass $M$ given by (e.g. LRS) 
$R_{0} \equiv \xi_{\mathrm{s}}\left(\frac{M}{4 \pi \xi_{\mathrm{s}}{ }^{2}\left|\theta_{\mathrm{s}}^{\prime}\right|}\right)^{\frac{1-n}{3-n}}\left[\frac{(n+1) K}{4 \pi G}\right]^{\frac{n}{3-n}}$,

and

$\kappa_{n} \equiv \frac{5}{3} \frac{\int_{0}^{\xi_{1}} \omega^{n} \xi^{4} d \xi}{\xi_{\mathrm{s}}^{4}\left|\omega_{\mathrm{s}}^{\prime}\right|}$,

where the prime indicates a derivative with respect to $\xi$, whilst the subscript $\mathrm{s}$ denotes a quantity evaluated at the surface. For a polytrope with index $n=1, \kappa_{n}=0.65345$ (LRS).

The ratio of kinetic to potential energy,

$\frac{T}{|W|} \equiv \frac{\tilde{T}+\frac{1}{2} \tilde{I} \tilde{\Omega}^{2}}{|\tilde{W}|}$,

is also compared to that of LRS.

For a polytropic system, the pressure $p$ is related to the density $\rho$ by equation (15). If we change variables to

$\xi=r \sqrt{\frac{4 \pi G}{(n+1) K} \rho_{\mathrm{c}}{ }^{1-\frac{1}{n}}}, \quad \omega=\left(\frac{\rho}{\rho_{\mathrm{c}}}\right)^{\frac{1}{n}}$,

the hydrostatic equation in spherical coordinates becomes the Lane-Emden equation (Shapiro \& Teukolsky 1983)

$\frac{1}{\xi^{2}} \frac{d}{d \xi}\left(\xi^{2} \frac{d \omega}{d \xi}\right)=-\omega^{n}$,

with central boundary conditions

$\omega(0)=1,\left.\quad \frac{d \omega}{d \xi}\right|_{\xi=0}=0$.

As the polytropic index, $n$, increases, so does the radius of the polytrope (which is defined as the position where the density drops to zero). For $n<5$ the radius is finite, whilst for $n=5$ the radius extends to infinity but the total mass remains finite. For $n>5$ the density drops off so slowly that both the mass and radius are infinite. The Lane-Emden equation (equation 62) has analytical solutions for $n=1,5$ (Binney \& Tremaine 1987). The case $n=1$ has the solution

$\omega=\frac{\sin \xi}{\xi}$.

This is one of the values for the polytropic index considered in both the calculations of LRS and Uryū \& Eriguchi (1998). Other values for the polytropic index of interest are $n=$ $1.5,3$. These polytropic indices are reasonable representations for the internal structure of convective and radiative stars, respectively. Table 1 gives the values of $\xi$ at the surface of the polytrope $\left(\xi_{\mathrm{s}}\right)$ and the ratio of mean density to central density $\left(\bar{\rho} / \rho_{\mathrm{c}}\right)$ for various polytropic indices (Kippenhahn \& Weigert 1994).

In our tests we considered the case $n=1$. This is the only analytical solution which has a finite radius making it a convenient starting approximation. Starting from this analytic solution we have calculated a compressible Maclaurin sequence for comparison, where we use 50 grid zones in the $r$ direction and 48 grid zones in the $\theta$ direction. We increased the value of $\Omega$ in units of 0.1 and used a time step of $\delta \tilde{t}=0.01$ to find a converged solution. We also included a linear artificial viscous pressure to damp out oscillations.

Figure 6 represents a comparison to the calculations of LRS. It shows a plot of the dimensionless angular velocity

\begin{tabular}{lcc}
\hline$n$ & $\xi_{\mathrm{s}}$ & $\bar{\rho} / \rho_{\mathrm{c}}$ \\
\hline 1 & 3.14159 & $3.03963 \times 10^{-1}$ \\
1.5 & 3.65375 & $1.66925 \times 10^{-1}$ \\
3 & 6.89685 & $1.84561 \times 10^{-2}$ \\
5 & $\infty$ & 0.0 \\
\hline
\end{tabular}

Table 4. Values of $\xi$ at the surface of a polytrope and the ratio of mean density to central density for various polytropic indices $n$.

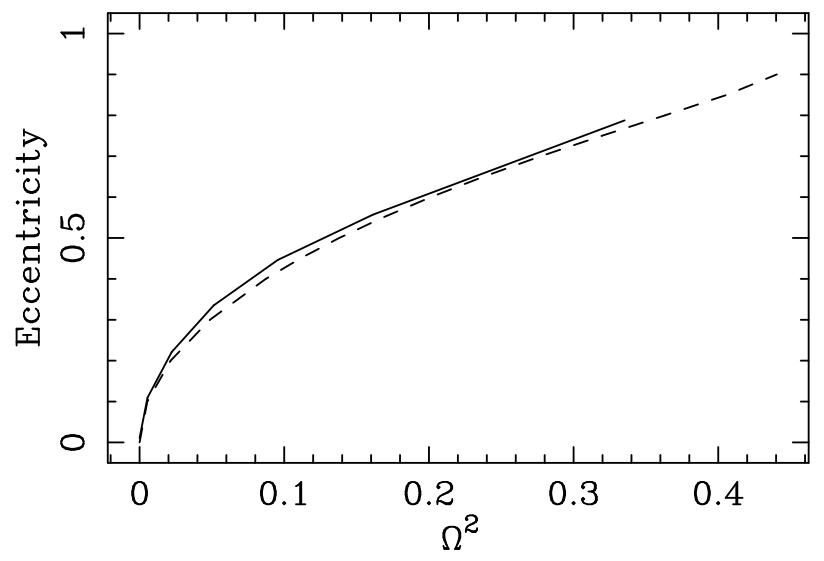

Figure 6. Angular velocity squared against eccentricity of an ellipsoid. The dashed curve represents the results of LRS and the solid curve our results for comparison.

squared against eccentricity. The figure shows that the code accurately calculates the eccentricity for each value of $\Omega$.

\section{FUTURE APPLICATIONS}

Our first application of the code will be the standard Roche problems. These have recently been considered by Uryu \& Eriguchi (1998), who assumed irrotational flows. We will apply our code to the same case for comparison and then relax the constraint of irrotation, using realistic surface boundary conditions. The second and main application will be the case of irradiation by X-rays in interacting binaries, where we will study the effects of surface heating, irradiation pressure and surface stresses to obtain self-consistent solutions of the geometry and the circulation system in irradiated systems and compare these results to observed systems where irradiation effects have been identified (e.g. HZ Her/Her X1, Cyg X-2, Nova Sco, Vela X-1, Sco X-1). At a later stage we will extend the code to more realistic stellar models as outlined in Appendix B.

\section{REFERENCES}

Barziv O., Kaper L., van Kerkwijk M. H., Telting J. H., van Paradijs J., 2001, A\&A, submitted (astro-ph/0108237)

Binney J., Tremaine S., Galactic Dynamics, Princeton, 1987

Casulli V., 1990, J. Comp. Phys., 86, 56

Casulli V., Cheng R. T., 1992, Int. J. Numer. Meth. Fluids, 15, 629

Davey S., Smith R. C., 1992, MNRAS, 257, 476 
Hunter C., 1962, ApJ, 136, 594

Kippenhahn R., Thomas H. C., 1979, A\&A, 75281

Kippenhahn R., Weigert A., Stellar Structure and Evolution, Springer, 1994

Kippenhahn R., Weigert A., Hofmeister E., 1967 in Alder B., Fernbach S., Rothenberg M., Methods in Computational Physics, Vol. 7, p. 129

Kırbıyık H., 1982, MNRAS, 200, 907

Kırbıyık H., Smith R. C., 1976, MNRAS, 176, 103

Lai D., Rasio F. A., Shapiro S. L., 1993, ApJS, 88, 205

Landau L. D., Lifshitz E. M., Course of Theoretical Physics, Vol. 6: Fluid Mechanics, Butterworth-Heinemann, 1987

LeVeque R. J., Mihilas D., Dorfi E. A., Müller E., Computational Methods for Astrophysical Fluid Flow, Springer, 1998

Lu Q., Wai O. W. H., 1998, Int. J. Numer. Meth. Fluids, 26, 771

Martin T. J., Davey S. C., 1995, MNRAS, 275, 31

Morse P. M., Feshbach H., Methods of Theoretical Physics, McGraw-Hill, 1953

Müller E., Steinmetz M., 1995, Comp. Phys. Comm., 89, 45

Orosz J. A., Bailyn C. D., 1997, ApJ, 477, 876

Pedlosky J., Geophysical Fluid Dynamics, Springer, 1987

Phillips S. N., 2000, D.Phil. Thesis, Oxford University, unpublished

Phillips S. N.. Podsiadlowski Ph., 2001, MNRAS submitted, (astro-ph/0109304)

Schandl S., Meyer-Hofmeister E., Meyer F., 1997, A\&A, 318, 73

Shahbaz T., Groot P., Phillips S. N., Casares J., Charles P. A., van Paradijs J., 2000, MNRAS, 314, 747

Shapiro S. L., Teukolsky S. A., 1983, Black Holes, White Dwarfs and Neutron Stars, Wiley

Stone J. M., Norman M. L., 1992, ApJS, 80, 753

Tassoul J.-L., 1978 Theory of Rotating Stars, Princeton

Tout C.A., Eggleton P.P., Fabian A.C., Pringle, J.E., 1989, MNRAS, 238, 427

Uryū K., Eriguchi Y., 1995, MNRAS, 277, 1411

Uryū K., Eriguchi Y., 1996, MNRAS, 282, 653

Uryū K., Eriguchi Y., 1998, ApJS, 118, 563

von Neumann J., Richtmeyer R. D., 1950, J. App. Phys., 21, 232

This paper has been typeset from a $\mathrm{T}_{\mathrm{E}} \mathrm{X} / \mathrm{L}_{\mathrm{E}} \mathrm{T} \mathrm{X}$ file prepared by the author. 


\section{APPENDIX A: SYSTEM VARIABLES}

Following are a list of variables used in this paper and a description of what they represent:

\begin{tabular}{|c|c|}
\hline$\frac{d}{d t}$ & Lagrangian derivative \\
\hline$\frac{\partial}{\partial t}$ & Eulerian derivative \\
\hline $\begin{array}{l}\partial t \\
p\end{array}$ & Pressure \\
\hline$\rho$ & Density \\
\hline $\mathcal{F}$ & Frictional force \\
\hline$u$ & Velocity in the rotating frame \\
\hline $\boldsymbol{u}^{a}$ & Velocity after the solution of the advective terms in the equation of motion (section 5.1) \\
\hline$\Omega$ & Angular velocity of rotation \\
\hline$\Phi$ & Potential \\
\hline$G$ & Gravitational constant \\
\hline$g$ & Surface gravity \\
\hline$r, \theta, \phi$ & Spherical Polar coordinates \\
\hline$A$ & Separation of the binary \\
\hline$r_{2}$ & Distance from the primary to the point $(r, \theta, \phi)$ \\
\hline$M_{1}$ & Mass of the primary \\
\hline$M_{2}$ & Mass of the secondary \\
\hline$\hat{n}$ & Unit surface normal vector \\
\hline$\hat{\boldsymbol{r}}, \hat{\boldsymbol{\theta}}, \hat{\boldsymbol{\phi}}$ & Unit vectors in the spherical coordinate system \\
\hline$K, n$ & Constants in the polytropic equation of state (equation 15) \\
\hline & Superscript denoting dimensionless variable (section 3.4) \\
\hline$N_{\mathrm{r}}, N_{\theta}, N_{\phi}$ & Number of $r, \theta, \phi$ elements \\
\hline $\mathrm{i}, \mathrm{j}, \mathrm{k}$ & Subscripts used to denote $r, \theta, \phi$ elements \\
\hline$\tau$ & Local optical depth in the secondary \\
\hline$\kappa$ & Local value of the opacity \\
\hline$L_{\mathrm{x}}$ & X-ray luminosity of the compact object \\
\hline$q$ & Artificial viscous pressure \\
\hline$C_{1}$ & Constant of order unity used in the calculation of artificial viscous pressure \\
\hline$C_{a}$ & The adiabatic speed of sound \\
\hline$\xi, \omega$ & Variables in the Lane-Emden equation for a polytrope (section 7) \\
\hline$V$ & Volume of the secondary \\
\hline$I$ & Moment of inertia of the secondary \\
\hline$J$ & Angular momentum of the system \\
\hline$T$ & Kinetic energy of the secondary \\
\hline$W$ & Potential energy of the secondary \\
\hline$\hat{\imath}$ & Superscript denoting universal dimensionless variable with no dependence on polytropic index (section 7) \\
\hline $\bar{R}$ & Mean radius of the secondary \\
\hline$R_{\text {pole }}$ & Polar radius of an ellipsoid \\
\hline$R_{\text {equator }}$ & Equatorial radius of an ellipsoid \\
\hline$R_{0}$ & Radius of a spherical polytrope of mass equal to the secondary \\
\hline
\end{tabular}

\section{APPENDIX B: GENERALISATION TO A NON-POLYTROPE}

In this appendix we describe how the computer code may be extended to more realistic stellar models. The most important change is to use a realistic (non-polytropic) equation of state and the addition of an energy equation, the thermodynamic equation (Tassoul 1978):

$\rho \frac{d U}{d t}+p \boldsymbol{\nabla} \cdot \boldsymbol{u}=\Phi_{\nu}+\rho \epsilon_{\mathrm{nuc}}-\nabla \cdot\left(\boldsymbol{F}+\boldsymbol{F}_{\mathbf{r}}\right)$

where $U$ is the total internal energy per unit mass, $\Phi_{\nu}$ the heat generation by viscous friction, $\epsilon_{\text {nuc }}$ the rate of energy released by thermonuclear reactions per unit mass, $\boldsymbol{F}$ the heat flux,

$\boldsymbol{F}=-\chi \nabla T$,

and $\boldsymbol{F}_{\mathbf{r}}$ is the radiative flux,

$\boldsymbol{F}_{\mathbf{r}}=-\frac{4 a c T^{3}}{3 \kappa \rho} \nabla T$,

where $a$ is the radiation constant and $T$ the temperature. The thermodynamic equation may also be written as 
$\rho T \frac{d S}{d t}=\Phi_{\nu}+\rho \epsilon_{\mathrm{nuc}}-\nabla \cdot\left(\boldsymbol{F}+\boldsymbol{F}_{\mathbf{r}}\right)$

where $S$ is the entropy per unit mass. Landau \& Lifshitz (1987) show that

$\frac{\partial S}{\partial t}=\left(\frac{\partial S}{\partial t}\right)_{p} \frac{\partial T}{\partial t}, \quad \nabla S=\left(\frac{\partial S}{\partial t}\right)_{p} \nabla T$.

Therefore,

$T \frac{d S}{d t}=c_{\mathrm{p}} \frac{d T}{d t}$

where $c_{\mathrm{p}}$ is the specific heat at constant pressure. This yields

$\rho c_{\mathrm{p}} \frac{d T}{d t}=\Phi_{\nu}+\rho \epsilon_{\mathrm{nuc}}-\nabla \cdot\left(\boldsymbol{F}+\boldsymbol{F}_{\mathbf{r}}\right)$

To relate pressure, density and temperature, we need an equation of state for the fluid, the simplest of which is given by the ideal gas law,

$p=\frac{k_{\mathrm{B}}}{\mu m_{\mathrm{H}}} \rho T$,

where $k_{\mathrm{B}}$ is Boltzmann's constant, $\mu$ the mean molecular weight and $m_{\mathrm{H}}$ the mass of the hydrogen atom.

The energy generation rate per unit mass, and the opacity are often represented as power laws of temperature and density (Uryū \& Eriguchi 1995), i.e.

$\epsilon_{\text {nuc }}=\epsilon_{0} \rho^{\gamma} T^{\delta}$

$\kappa=\kappa_{0} \rho^{\alpha} T^{-\beta}$,

where $\epsilon_{0}, \gamma, \delta, \kappa_{0}, \alpha$ and $\beta$ are constants. For simplified models for lower main-sequence stars, appropriate values for these constants are

$\gamma \simeq 1, \quad \delta \simeq 4.5$

and for Kramers' opacity law,

$\alpha=1, \quad \beta=3.5$.

Inclusion of the thermodynamic equation introduces temperature as another variable into the problem along with other variables which depend on $p, \rho, \boldsymbol{u}$ and $T$. This leads altogether to six equations and six unknowns. It also requires an additional surface boundary condition, which can be determined from an atmosphere calculation where the effects of external irradiation (if present) may also be included, just as in the case for standard stellar-structure calculations (e.g. Kippenhahn, Weigert \& Hofmeister 1967). These equations have to be solved simultaneously to yield the solution at each time step.

\section{APPENDIX C: THE CALCULATION OF THE POTENTIAL}

In this appendix we derive analytical formulae for the derivatives of the gradient of the potential and describe the technique used in their implementation.

\section{C1 The derivatives of the potential}

Differentiating equations (30) to (32) with respect to $r$, we obtain:

$$
\begin{aligned}
& \frac{\partial \Phi(r, \theta, \phi)}{\partial r}=-G \sum_{l=0}^{\infty} \frac{4 \pi}{2 l+1} \sum_{m=-l}^{l} Y^{l m}(\theta, \phi)\left[\frac{-(l+1)}{r^{l+2}} C^{l m}(r)+\frac{1}{r^{l+1}} \frac{\partial C^{l m}(r)}{\partial r}+l r^{l-1} D^{l m}(r)+r^{l} \frac{\partial D^{l m}(r)}{\partial r}\right] . \\
& \frac{\partial C^{l m}(r)}{\partial r}=r^{l+2} \int_{4 \pi} d \Omega^{\prime} Y^{l m *}\left(\theta^{\prime}, \phi^{\prime}\right) \rho\left(r^{\prime}, \theta^{\prime}, \phi^{\prime}\right) \\
& \frac{\partial D^{l m}(r)}{\partial r}=-r^{1-l} \int_{4 \pi} d \Omega^{\prime} Y^{l m *}\left(\theta^{\prime}, \phi^{\prime}\right) \rho\left(r^{\prime}, \theta^{\prime}, \phi^{\prime}\right) .
\end{aligned}
$$

Hence,

$\frac{1}{r^{l+1}} \frac{\partial C^{l m}(r)}{\partial r}+r^{l} \frac{\partial D^{l m}(r)}{\partial r}=0$,

and the $r$ derivative of the potential becomes

(C) 0000 RAS, MNRAS 000, 000-000 
$\frac{\partial \Phi(r, \theta, \phi)}{\partial r}=-G \sum_{l=0}^{\infty} \frac{4 \pi}{2 l+1} \sum_{m=-l}^{l} Y^{l m}(\theta, \phi)\left[\frac{-(l+1)}{r^{l+2}} C^{l m}(r)+l r^{l-1} D^{l m}(r)\right]$.

The $\theta$ derivative is given by

$\frac{\partial \Phi(r, \theta, \phi)}{\partial \theta}=-G \sum_{l=0}^{\infty} \frac{4 \pi}{2 l+1} \sum_{m=-l}^{l} \frac{\partial Y^{l m}(\theta, \phi)}{\partial \theta}\left[\frac{1}{r^{l+1}} C^{l m}(r)+r^{l} D^{l m}(r)\right]$.

Using the definition of the spherical harmonics, we find

$\frac{\partial \Phi(r, \theta, \phi)}{\partial \theta}=-G \sum_{l=0}^{\infty} \frac{4 \pi}{2 l+1} \sum_{m=-l}^{l} \frac{Y^{l m}(\theta, \phi)}{P_{l}^{|m|}(\cos \theta)} \frac{d\left[P_{l}^{m}(\cos \theta)\right]}{d \theta}\left[\frac{1}{r^{l+1}} C^{l m}(r)+r^{l} D^{l m}(r)\right]$.

Using one of the recurrence relations for Legendre polynomials,

$P_{l}^{m}(\cos \theta)=(-1)^{m} \sin ^{m} \theta \frac{d^{m}\left[P_{l}(\cos \theta)\right]}{d(\cos \theta)^{m}}$,

we then obtain

$$
\begin{aligned}
P_{l}^{m+1}(\cos \theta) & =(-1)^{m+1} \sin ^{m+1} \theta \frac{d^{m+1}\left[P_{l}(\cos \theta)\right]}{d(\cos \theta)^{m+1}}, \\
& =(-1)^{m+1} \sin ^{m+1} \theta\left(\frac{-1}{\sin \theta}\right) \frac{d}{d \theta}\left[\frac{P_{l}^{m}(\cos \theta)}{(-1)^{m} \sin ^{m} \theta}\right], \\
& =\frac{d\left[P_{l}^{m}(\cos \theta)\right]}{d \theta}-P_{l}^{m}(\cos \theta)\left(\frac{m \cos \theta \sin ^{m-1} \theta}{\sin ^{m} \theta}\right),
\end{aligned}
$$

Rearranging these, we get the required relation

$\frac{d\left[P_{l}^{m}(\cos \theta)\right]}{d \theta}=P_{l}^{m+1}(\cos \theta)+\frac{m \cos \theta}{\sin \theta} P_{l}{ }^{m}(\cos \theta)$.

When $m=l$, we set $P_{l}^{m+1}$ equal to zero. The $\theta$ derivative is then

$$
\frac{\partial \Phi(r, \theta, \phi)}{\partial \theta}=-G \sum_{l=0}^{\infty} \frac{4 \pi}{2 l+1} \sum_{m=-l}^{l} Y^{l m}(\theta, \phi)\left[\frac{P_{l}^{|m+1|}(\cos \theta)}{P_{l}^{|m|}(\cos \theta)}+\frac{m \cos \theta}{\sin \theta}\right]\left[\frac{1}{r^{l+1}} C^{l m}(r)+r^{l} D^{l m}(r)\right] \text {. }
$$

Finally, the $\phi$ derivative is simply given by

$$
\begin{aligned}
\frac{\partial \Phi(r, \theta, \phi)}{\partial \phi} & =-G \sum_{l=0}^{\infty} \frac{4 \pi}{2 l+1} \sum_{m=-l}^{l} \frac{\partial Y^{l m}(\theta, \phi)}{\partial \phi}\left[\frac{1}{r^{l+1}} C^{l m}(r)+r^{l} D^{l m}(r)\right] \\
& =-G \sum_{l=0}^{\infty} \frac{4 \pi}{2 l+1} \sum_{m=-l}^{l} i m Y^{l m}(\theta, \phi)\left[\frac{1}{r^{l+1}} C^{l m}(r)+r^{l} D^{l m}(r)\right]
\end{aligned}
$$

\section{C2 Implementation}

To implement the expansion of the potential in spherical harmonics, we follow the technique described in Müller \& Steinmetz (1995), in which the integrals are split into a sum of integrals over sub-intervals. If we denote the position at which equations (31) \& (32) are to be evaluated as $r_{\mathrm{n}}, C^{l m}(r)$ as $C_{\mathrm{n}}^{l m}$, and $D^{l m}(r)$ as $D_{\mathrm{n}}^{l m}$ :

$\begin{aligned} C_{\mathrm{n}}^{l m} & =\sum_{\mathrm{j}=1}^{N_{\theta}} \sum_{\mathrm{k}=1}^{N_{\phi}}\left[\int_{\phi_{\mathrm{k}-1}}^{\phi_{\mathrm{k}}} \int_{\theta_{\mathrm{j}-1}}^{\theta_{\mathrm{j}}} \sin \theta d \theta d \phi Y^{l m *}(\theta, \phi) \sum_{\mathrm{i}=1}^{n} \int_{r_{\mathrm{i}-1}}^{r_{\mathrm{i}}} d r r^{l+2} \rho(r, \theta, \phi)\right], \\ D_{\mathrm{n}}^{l m} & =\sum_{\mathrm{j}=1}^{N_{\theta}} \sum_{\mathrm{k}=1}^{N_{\phi}}\left[\int_{\phi_{\mathrm{k}-1}}^{\phi_{\mathrm{k}}} \int_{\theta_{\mathrm{j}-1}}^{\theta_{\mathrm{j}}} \sin \theta d \theta d \phi Y^{l m *}(\theta, \phi) \sum_{\mathrm{i}=\mathrm{n}+1}^{N_{\mathrm{r}}} \int_{r_{\mathrm{i}-1}}^{r_{\mathrm{i}}} d r r^{1-l} \rho(r, \theta, \phi)\right] .\end{aligned}$

Introducing $A_{\mathrm{i}, \mathrm{j}, \mathrm{k}}^{l m}$ and $B_{\mathrm{i}, \mathrm{j}, \mathrm{k}}^{l m}$,

$A_{\mathrm{i}, \mathrm{j}, \mathrm{k}}^{l m}=\int_{\phi_{\mathrm{k}-1}}^{\phi_{\mathrm{k}}} \int_{\theta_{\mathrm{j}-1}}^{\theta_{\mathrm{j}}} \int_{r_{\mathrm{i}-1}}^{r_{\mathrm{i}}} \sin \theta d \theta d \phi d r r^{l+2} Y^{l m *}(\theta, \phi) \rho(r, \theta, \phi)$,

$B_{\mathrm{i}, \mathrm{j}, \mathrm{k}}^{l m}=\int_{\phi_{\mathrm{k}-1}}^{\phi_{\mathrm{k}}} \int_{\theta_{\mathrm{j}-1}}^{\theta_{\mathrm{j}}} \int_{r_{\mathrm{i}-1}}^{r_{\mathrm{i}}} \sin \theta d \theta d \phi d r r^{1-l} Y^{l m *}(\theta, \phi) \rho(r, \theta, \phi)$, 
we may write

$C_{\mathrm{n}}^{l m}=\sum_{\mathrm{i}=1}^{n} \sum_{\mathrm{j}=1}^{N_{\theta}} \sum_{\mathrm{k}=1}^{N_{\phi}} A_{\mathrm{i}, \mathrm{j}, \mathrm{k}}^{l m}=C_{n-1}^{l m}+\sum_{\mathrm{j}=1}^{N_{\theta}} \sum_{\mathrm{k}=1}^{N_{\phi}} A_{\mathrm{n}, \mathrm{j}, \mathrm{k}}^{l m}$,
$D_{\mathrm{n}}^{l m}=\sum_{\mathrm{i}=\mathrm{n}+1}^{N_{\mathrm{r}}} \sum_{\mathrm{j}=1}^{N_{\theta}} \sum_{\mathrm{k}=1}^{N_{\phi}} B_{\mathrm{i}, \mathrm{j}, \mathrm{k}}^{l m}=D_{n+1}^{l m}+\sum_{\mathrm{j}=1}^{N_{\theta}} \sum_{\mathrm{k}=1}^{N_{\phi}} B_{\mathrm{n}+1, \mathrm{j}, \mathrm{k}}^{l m}$.

We implement this as

$A_{\mathrm{i}, \mathrm{j}, \mathrm{k}}^{l m}=\sin \theta_{\mathrm{j}} \delta \theta \delta \phi Y^{l m *}\left(\theta_{\mathrm{j}}, \phi_{\mathrm{k}}\right) \int_{r_{\mathrm{i}-1}}^{r_{\mathrm{i}}} \rho\left(r, \theta_{\mathrm{j}}, \phi_{\mathrm{k}}\right) r^{l+2} d r$

$B_{\mathrm{i}, \mathrm{j}, \mathrm{k}}^{l m}=\sin \theta_{\mathrm{j}} \delta \theta \delta \phi Y^{l m *}\left(\theta_{\mathrm{j}}, \phi_{\mathrm{k}}\right) \int_{r_{\mathrm{i}-1}}^{r_{\mathrm{i}}} \rho\left(r, \theta_{\mathrm{j}}, \phi_{\mathrm{k}}\right) r^{1-l} d r$

For the radial integration we assume that the density varies linearly with $r$ within each cell

$$
\begin{aligned}
\rho\left(r, \theta_{\mathrm{j}}, \phi_{\mathrm{k}}\right) & =\rho\left(r_{\mathrm{i}-1}, \theta_{\mathrm{j}}, \phi_{\mathrm{k}}\right)+\frac{\left(r-r_{\mathrm{i}-1}\right) \delta \rho}{\left(r_{\mathrm{i}}-r_{\mathrm{i}-1}\right)}, \\
& =\rho_{\mathrm{i}-1, \mathrm{j}, \mathrm{k}}+\frac{\left(r-r_{\mathrm{i}-1}\right) \delta \rho}{\left(r_{\mathrm{i}}-r_{\mathrm{i}-1}\right)},
\end{aligned}
$$

where

$\delta \rho=\rho_{\mathrm{i}, \mathrm{j}, \mathrm{k}}-\rho_{\mathrm{i}-1, \mathrm{j}, \mathrm{k}}$.

Hence

$$
\begin{aligned}
& A_{\mathrm{i}, \mathrm{j}, \mathrm{k}}^{l m}=\sin \theta_{\mathrm{j}} \delta \theta \delta \phi Y^{l m *}\left(\theta_{\mathrm{j}}, \phi_{\mathrm{k}}\right)\left[\left(\rho_{\mathrm{i}-1, \mathrm{j}, \mathrm{k}}-\frac{r_{i-1} \delta \rho}{r_{\mathrm{i}}-r_{\mathrm{i}-1}}\right) \frac{r^{l+3}}{l+3}+\frac{\delta \rho}{\left(r_{\mathrm{i}}-r_{\mathrm{i}-1}\right)} \frac{r^{l+4}}{l+4}\right]_{r_{\mathrm{i}}-1}^{r_{\mathrm{i}}},
\end{aligned}
$$

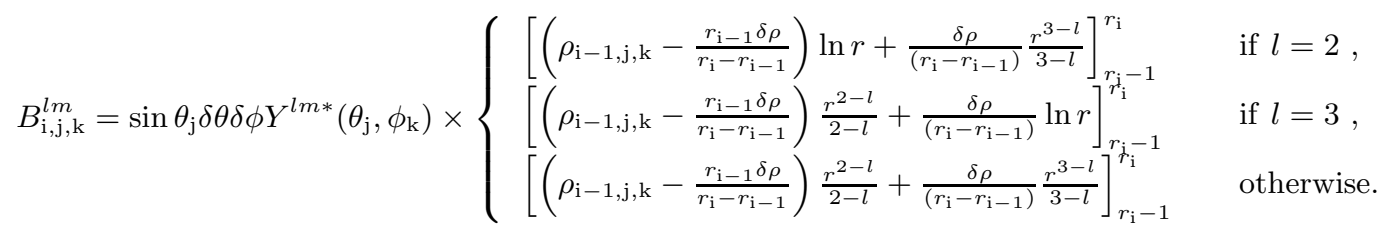

The $r$ derivative of the potential is evaluated at the radial boundary of a cell and the $\theta$ and $\phi$ derivatives at the radial center of a cell. Hence, in the code, two sets of $C^{l m}(r)$ and $D^{l m}(r)$ are calculated: one for the $r$ derivatives and the other for the $\theta$ and $\phi$ derivatives.

In our calculations we consider spherical harmonics up to and including the $l=14$ term. This choice follows from the results presented by Müller \& Steinmetz (1995) who find (as we do) that the inclusion of terms of higher order does not significantly affect the calculation for geometries in which the use of spherical coordinates is appropriate.

\section{APPENDIX D: FINITE DIFFERENCE FORM OF EQUATIONS}

In this appendix we give the finite difference form for the terms used in equations (9) \& (14). As velocities are calculated on cell boundaries, and pressure and density at the center of cells, it is simple to represent the pressure term (from equation 9) in finite difference form as

$\frac{1}{\rho} \frac{\partial p}{\partial r}=\frac{2 N_{\mathrm{r}}\left(p_{\mathrm{i}+1, \mathrm{j}, \mathrm{k}}-p_{\mathrm{i}, \mathrm{j}, \mathrm{k}}\right)}{R_{\mathrm{j}, \mathrm{k}}\left(\rho_{\mathrm{i}+1, \mathrm{j}, \mathrm{k}}+\rho_{\mathrm{i}, \mathrm{j}, \mathrm{k}}\right)}$

and similarly for the derivatives in the $\theta$ and $\phi$ directions. The Coriolis term in the equation of motion is solved using equation (42). With the notation $u, v, w$ to represent $u_{\mathrm{r}}, u_{\theta}, u_{\phi}$ for ease of reading, the three components of equation (42) are

$\frac{u_{\mathrm{i}, \mathrm{j}, \mathrm{k}}^{a}-u_{\mathrm{p}_{\mathrm{i}, \mathrm{j}, \mathrm{k}}}^{n}}{\delta t}=2 \Omega w_{\mathrm{i}+\frac{1}{2}, \mathrm{j}, \mathrm{k}+\frac{1}{2}}^{a} \sin \theta_{j}$,

$\frac{v_{\mathrm{i}, \mathrm{j}, \mathrm{k}}^{a}-v_{\mathrm{p}_{\mathrm{i}, \mathrm{j}, \mathrm{k}}}^{n}}{\delta t}=2 \Omega w_{\mathrm{i}, \mathrm{j}-\frac{1}{2}, \mathrm{k}+\frac{1}{2}}^{a} \cos \theta_{j-\frac{1}{2}}$

$\frac{w_{\mathrm{i}, \mathrm{j}, \mathrm{k}}^{a}-w_{\mathrm{p}_{\mathrm{i}, \mathrm{j}, \mathrm{k}}}^{n}}{\delta t}=-2 \Omega\left(u_{\mathrm{i}-\frac{1}{2}, \mathrm{j}, \mathrm{k}-\frac{1}{2}}^{a} \sin \theta_{j}+v_{\mathrm{i}, \mathrm{j}+\frac{1}{2}, \mathrm{k}-\frac{1}{2}}^{a} \cos \theta_{j}\right)$. 


\section{M. E. Beer and Ph. Podsiadlowski}

The integral form of the continuity equation (14) in finite difference form is

$$
\begin{aligned}
& \frac{\rho_{\mathrm{i}, \mathrm{j}, \mathrm{k}}^{n+1}-\rho_{\mathrm{i}, \mathrm{j}, \mathrm{k}}^{n}}{\delta t} \delta\left(\frac{r^{3}}{3}\right) \delta(-\cos \theta) \delta \phi+\rho_{\mathrm{i}+\frac{1}{2}, \mathrm{j}, \mathrm{k}}^{n+\frac{1}{2}}\left(u_{\mathrm{i}, \mathrm{j}, \mathrm{k}} \hat{\boldsymbol{n}} \cdot \hat{\boldsymbol{r}}+v_{\mathrm{i}+\frac{1}{2}, \mathrm{j}+\frac{1}{2}, \mathrm{k}} \hat{\boldsymbol{n}} \cdot \hat{\boldsymbol{\theta}}+w_{\mathrm{i}+\frac{1}{2}, \mathrm{j}, \mathrm{k}+\frac{1}{2}} \hat{\boldsymbol{n}} \cdot \hat{\boldsymbol{\phi}}\right) r_{\mathrm{i}+\frac{1}{2}, \mathrm{j}, \mathrm{k}}^{2} \delta \phi \delta(-\cos \theta)- \\
& \quad \rho_{\mathrm{i}-\frac{1}{2}, \mathrm{j}, \mathrm{k}}^{n+\frac{1}{2}} \times\left(u_{\mathrm{i}-1, \mathrm{j}, \mathrm{k}} \hat{\boldsymbol{n}} \cdot \hat{\boldsymbol{r}}+v_{\mathrm{i}-\frac{1}{2}, \mathrm{j}+\frac{1}{2}, \mathrm{k}} \hat{\boldsymbol{n}} \cdot \hat{\boldsymbol{\theta}}+w_{\mathrm{i}-\frac{1}{2}, \mathrm{j}, \mathrm{k}+\frac{1}{2}} \hat{\boldsymbol{n}} \cdot \hat{\boldsymbol{\phi}}\right) r_{\mathrm{i}-\frac{1}{2}, \mathrm{j}, \mathrm{k}}^{2} \delta \phi \delta(-\cos \theta)+\rho_{\mathrm{i}, \mathrm{j}+\frac{1}{2}, \mathrm{k}}^{n+\frac{1}{2}} v_{\mathrm{i}, \mathrm{j}+1, \mathrm{k}} \sin \theta_{j+\frac{1}{2}} r_{\mathrm{i}, \mathrm{j}+\frac{1}{2}, \mathrm{k}} \delta r \delta \phi \\
& \quad-\rho_{\mathrm{i}, \mathrm{j}-\frac{1}{2}, \mathrm{k}}^{n+\frac{1}{2}} v_{\mathrm{i}, \mathrm{j}, \mathrm{k}} \sin \theta_{j-\frac{1}{2}} r_{\mathrm{i}, \mathrm{j}-\frac{1}{2}, \mathrm{k}} \delta r \delta \phi+\rho_{\mathrm{i}, \mathrm{j}, \mathrm{k}+\frac{1}{2}}^{n+\frac{1}{2}} w_{\mathrm{i}, \mathrm{j}, \mathrm{k}+1} r_{\mathrm{i}, \mathrm{j}, \mathrm{k}+\frac{1}{2}} \delta r \delta(-\cos \theta)-\rho_{\mathrm{i}, \mathrm{j}, \mathrm{k}-\frac{1}{2}}^{n+\frac{1}{2}} w_{\mathrm{i}, \mathrm{j}, \mathrm{k}} r_{\mathrm{i}, \mathrm{j}, \mathrm{k}-\frac{1}{2}} \delta r \delta(-\cos \theta)=0 .
\end{aligned}
$$

